\title{
Ground-state self-consistent calculation of quantum dots under magnetic fields: Addition spectrum
}

\author{
N. Barberan \\ Departament d'Estructura i Constituents de la Matèria, Facultat de Física, Universitat de Barcelona, E-08028 Barcelona, Spain
}

(Received 7 May 1998; revised manuscript received 16 July 1998)

\begin{abstract}
A density-functional self-consistent calculation of the ground-state electronic density of quantum dots under an arbitrary magnetic field is performed. We consider a parabolic lateral confining potential. The addition energy, $E(N+1)-E(N)$, where $N$ is the number of electrons, is compared with experimental data and the different contributions to the energy are analyzed. The Hamiltonian is modeled by a density functional, which includes the exchange and correlation interactions and the local formation of Landau levels for different equilibrium spin populations. We obtain an analytical expression for the critical density under which spontaneous polarization, induced by the exchange interaction, takes place. [S0163-1829(98)07740-6]
\end{abstract}

\section{INTRODUCTION}

Quantum dots represent systems in which the transition from quantum to semiclassical physics can be tested in an increasingly controlled way and are suitable for comparing different theoretical approaches with experimental data. ${ }^{1}$ Here we concentrate on the study of the ground state (g.s.) properties. We examine the formation of compressible and incompressible regions within the dot and their dependence on the applied magnetic field as well as the different contributions to the energy from the different terms of the Hamiltonian. Finally, we propose an interpretation of the addition spectrum in the quantum Hall regime given by McEuen et $a .^{2}$

In order to develop a managable method for dealing with a wide range of quantum dots, an alternative model to the Thomas-Fermi calculation given by McEuen et al. ${ }^{2}$ or Marmorkos and Beenakker ${ }^{3}$ has been developed. There are two main differences between our model and the standard Thomas-Fermi or semiclassical approaches previously reported in the literature (see, for example, Refs. 2, 4, 5, and 6):

(1) The kinetic term in the Hamiltonian is given by a local density functional for each spin population, taking into account the Landau Levels (LL) created by the magnetic field. In Ref. 2 the kinetic contribution is not given by a local term, but instead each LL energy is multiplied by a global density $\rho_{n s}$ (within the whole dot) assigned to this level, characterized by the $n s$ indexes, and self-consistently calculated. The level densities are treated separately on the assumption that the coupling between states in distinct LL's is small. Our treatment produces total charges for each LL that are not restricted to integer numbers and therefore, compatible with the idea of the formation of "melting states" as discussed by Palacios et al. $^{7}$

(2) The exchange interaction is taken into account in two different ways: as a density functional term in the Hamiltonian and as an interaction that determines (together with the kinetic term and spin-magnetic field interaction) the spin populations that locally minimize the energy. In McEuen's paper the exchange interaction is not taken into account at all. We obtain an analytical expression for the critical density under which the system is spontaneously fully polarized.

As in McEuen's work, ours is a self-consistent calculation, and benefits from the ability to analyze each contribution of the Hamiltonian directly.

This paper is organized as follows. In Sec. II we describe the model used in our calculation, the different parts of the Hamiltonian are described, with special emphasis being placed on the kinetic term and on the way in which the spin population is locally determined. In Sec. III the numerical method used in the iterative calculation is described and in Sec. IV detailed numerical results for electronic densities and g.s. energies for various magnetic fields are presented. We propose a possible interpretation of the addition spectra.

\section{THE MODEL USED}

The total energy of the system is given by the twodimensional (2D) integral,

$$
E_{\mathrm{tot}}=\int \overrightarrow{d r}\left[\epsilon_{k}+\epsilon_{\mathrm{conf}}+\epsilon_{\mathrm{dir}}+\epsilon_{\mathrm{ex}}+\epsilon_{\mathrm{cor}}+\epsilon_{B}\right],
$$

where in the kinetic term

$$
\epsilon_{k}=\epsilon_{k 1}+\epsilon_{k 2}
$$

the subindexes distinguish the different spin populations and

$$
\epsilon_{k i}=\frac{\hbar \omega_{c}}{2} D S_{i}^{2}+\hbar \omega_{c}\left(S_{i}+\frac{1}{2}\right)\left(n_{i}-S_{i} D\right), \quad i=1,2
$$

The first term is the contribution to the kinetic energy from the fully occupied LL, whereas the second term is the contribution that comes from the last, possible partially occupied LL. $D=B e / h c$ is the LL degeneracy per unit area, $B$ being the magnetic field strength, $e$ the electronic charge, and $c$ the light velocity in vacuum. Locally, we consider a free system of independent electrons under a magnetic field in a dot of area $\pi R^{2}$. The cyclotron frequency is given by $\omega_{c}$ $=e B / \mathrm{cm}^{*}, m^{*}$ being the effective electron mass, $S_{i}$ the last LL fully (locally) occupied and $n_{i}$ the electronic density given by 


$$
n_{1}=\frac{1}{2} n(1-\xi) \quad \text { or } \quad n_{2}=\frac{1}{2} n(1+\xi),
$$

where $0 \leqslant \xi \leqslant 1$ is the spin asymmetry.

$S_{i}$ is defined as

$$
S_{i}=\operatorname{Integer}\left[\frac{n_{i}(r)}{D}\right]
$$

The $\xi$ parameter is determined by minimizing the energy against $\xi$ as is explained below. The next term,

$$
\epsilon_{\mathrm{conf}}=\frac{1}{2} m^{*} \omega_{0}^{2} r^{2} n(r), \quad n=n_{1}+n_{2},
$$

is the contribution that comes from the confining potential, assumed to be parabolic (nonquadratic terms can be added to see the effects of nonparabolicity).

The next three terms in Eq. (1) come from the Coulomb interaction. The direct Coulomb term is given by

$$
\epsilon_{\mathrm{dir}}=\frac{e}{2} V(r) n
$$

where $V(r)$ is the potential created by the $2 \mathrm{D}$ electronic disk, and is given by

$$
\begin{aligned}
V(r)= & \frac{e}{\varepsilon} \int \frac{n\left(\overrightarrow{r^{\prime}}\right) d \overrightarrow{r^{\prime}}}{\left|\vec{r}-\overrightarrow{r^{\prime}}\right|}=\frac{12}{r} \frac{e}{\varepsilon} \int_{0}^{r} d x x n(x) E\left(\frac{x}{r}\right) \\
& +\frac{4}{r} \frac{e}{\varepsilon} \int_{0}^{r} d x x^{2} n^{\prime}(x) E\left(\frac{x}{r}\right) \\
& -4 \frac{e}{\varepsilon} \int_{r}^{\infty} d x x n^{\prime}(x) E\left(\frac{r}{x}\right)-8 r \frac{e}{\varepsilon} n(r),
\end{aligned}
$$

where $E$ is the complete elliptic integral of the second kind, ${ }^{8}$ and $\varepsilon$ is the dielectric constant of the semiconductor.

The exchange term is given by the expression

$$
\epsilon_{e x}=-\frac{8}{3 \sqrt{\pi}} \frac{e^{2}}{\varepsilon}\left(\frac{n}{2}\right)^{3 / 2}\left[(1-\xi)^{3 / 2}+(1+\xi)^{3 / 2}\right]
$$

calculated from the exchange energy term per electron in a 2D system given in Refs. 9 and 10 by

$$
E_{\mathrm{ex}}(\vec{k})=-\frac{2 e^{2}}{\pi \varepsilon} k_{F} E\left(\frac{k^{2}}{k_{F}^{2}}\right),
$$

once it is averaged and separately considered for each spin population.

Finally, the Zeeman splitting and correlation terms in Eq. (1) are given by

$$
\epsilon_{B}=\frac{g}{2} \mu_{B} B \xi n
$$

and

$$
\epsilon_{\mathrm{cor}}=-0.977 \frac{e^{2}}{\varepsilon}\left(\frac{n^{3 / 2}}{1+7.8165 a_{B}^{*} n^{1 / 2}}\right),
$$

where $g$ is the Lande factor appropriate to the semiconductor, $\mu_{B}$ is the Bohr magneton (for the free electron mass,

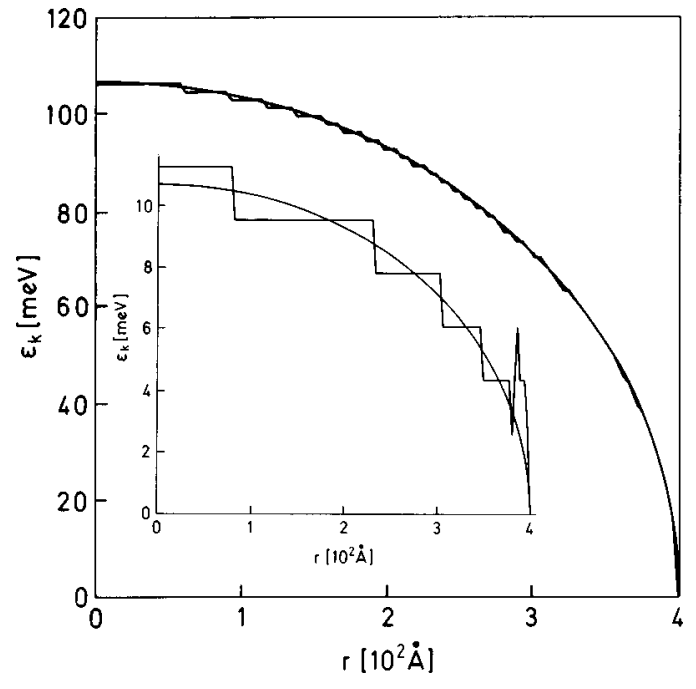

FIG. 1. Kinetic contribution to the Euler equation along the dot radius for a dome density. The smooth curve is the Thomas-Fermi result and the stepped curve the LL type result for $N=100$ and for $N=10$ in the inset. $B=1 \mathrm{~T}$ has been considered in both cases.

$\left.\mu_{B}=e \hbar / 2 m c\right)$, and $\epsilon_{\text {cor }}$ is the Wigner approximation calculated for a 2D system, ${ }^{11}$ where $a_{B}^{*}=\varepsilon \hbar^{2} / m^{*} e^{2}$.

By minimizing the total energy of the dot with respect to the density for a fixed number of electrons, an EulerLagrange equation is obtained,

$$
\frac{\partial \epsilon_{\mathrm{tot}}(n)}{\partial n}=\mu_{c}
$$

where $\mu_{c}$ is the chemical potential and $\epsilon_{\text {tot }}(n)$ is the density functional energy per unit area obtained from the six terms in Eq. (1).

It must be emphasized that each term is calculated within a local approximation and especially the kinetic term at $r, \epsilon_{k}(r)$ is the contribution of a 2D free-electron gas of density $n(r)$ under a magnetic field, in the same spirit as the Thomas-Fermi functional is calculated for an infinite homogeneous gas. For a large number of electrons, and low magnetic field, our kinetic functional approaches the 2D ThomasFermi contribution: $\hbar^{2} \pi n(r) / m^{*}$ as expected (see Fig. 1).

The local approximation is expected to be reasonably good, due to the partial cancellation between the direct Coulomb term and the confining potential, a cancellation that produces a rather flat effective potential within the dot area, as is verified below.

The functional variation of each of the six terms in Eq. (1) is straightforward, except in the case of the kinetic contribution where some care must be taken. To complete this section, we will discus the kinetic contribution to the Euler equation and the calculation of the $\xi$ parameter in the next two subsections.

\section{A. Kinetic contribution}

If one assumes that the addition of an extra electron (let us say in the spin up population) leaves the number of fully occupied LL's, $(S)$ unchanged and that only the highest partially filled level is affected, then the nonvanishing contribu- 


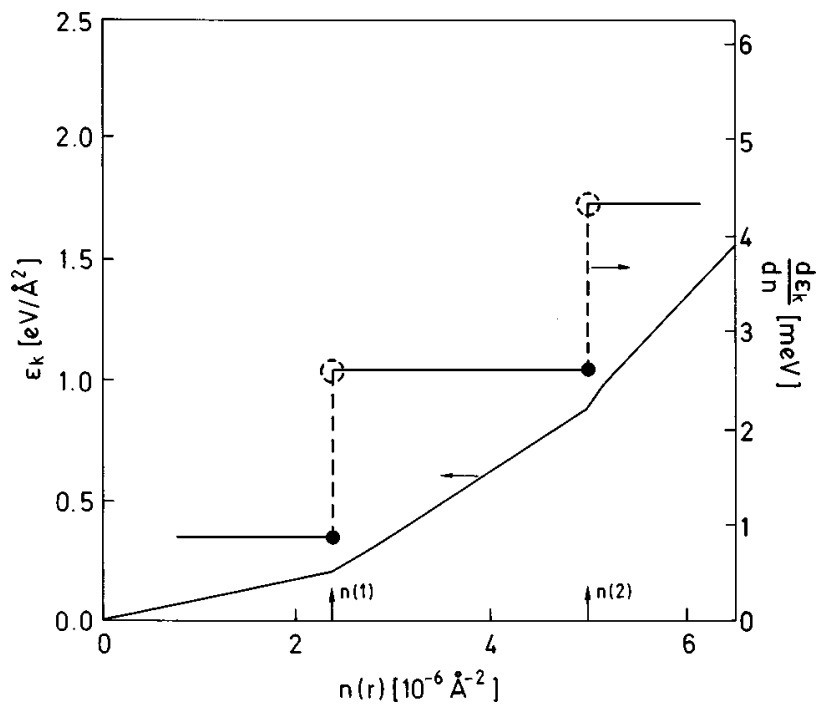

FIG. 2. Left axis labeling: Kinetic contribution to the energy density as a function of the electronic density. The arrows correspond to integer filling factors [at $n(i)]$. Right axis labeling: Kinetic contribution to the Euler equation. $B=1 \mathrm{~T}$ in both cases.

tion to $\partial \epsilon_{k} / \partial n$ comes from the $n_{i}$-dependent part of the second term on the right-hand side of Eq. (3) and is given by

$$
\hbar \omega_{c}\left(S_{i}+\frac{1}{2}\right)
$$

A subtle problem arises from those values of the local electronic density that produce an integer filling factor, due to the discontinuities of the function $\partial \epsilon_{k} / \partial n$ at these values of the density ${ }^{12}$ (see Fig. 2).

A simple way to solve the uncertainty at the points of discontinuity is given by the consideration that $\partial \epsilon_{k} / \partial n$ is the energy of an electron at the Fermi level (the highest possible single-particle state). From this point of view, the value of its energy, once the local density and the degeneracy $D$ are known is clear. The value that one must assign to $\partial \epsilon_{k} / \partial n$ at $n(i)$ (see Fig. 2) is the one given by the lower edge of the gap (the black dots in Fig. 2).

The final expresion for the Euler equation is then given as

$$
\begin{aligned}
\frac{\partial \epsilon_{t o t}}{\partial n}= & C+e V(r)+\frac{1}{2} m^{*} \omega_{0}^{2} r^{2}+\frac{g}{2} \mu_{B} B \\
& -\frac{e^{2}}{\varepsilon} 4\left[\frac{n}{2 \pi}(1+\xi)\right]^{1 / 2} \\
& -0.9775 \frac{e^{2}}{\varepsilon} \frac{\frac{3}{2} n^{1 / 2}+7.8165 a_{B}^{*} n}{\left(1+7.8165 a_{B}^{*} n^{1 / 2}\right)^{2}},
\end{aligned}
$$

where $C$ is given by

$$
C=\left\{\begin{array}{l}
0 \quad \text { if } \quad \alpha=0 \quad \text { and } S=0 \\
\hbar \omega_{c}\left(S-\frac{1}{2}\right) \quad \text { if } \quad \alpha=0 \text { and } S \neq 0 \\
\hbar \omega_{c}\left(S+\frac{1}{2}\right) \quad \text { if } \quad \alpha \neq 0,
\end{array}\right.
$$

$\alpha$ being the difference between $n(r) / D$ and $S$ and where an electron inside the greatest spin population $\left(S=S_{2}\right)$ has been considered, i.e., the derivative is taken with respect to $n_{2}$, keeping $n_{1}$ fixed.

For a system of electrons in the absence of magnetic field, the non-negative function $n(r)$ that produces a constant function on the right-hand side of Eq. (13) is the g.s. density. However, the presence of a magnetic field localizes the electrons in space and the functions involved in the Euler equation have to be redefined.

To reconcile the definition of the function $C$ given by Eq. (16), which has abrupt changes along $r$ that are only partially compensated by the Coulomb terms, with the presence of a constant chemical potential on the right-hand side of Eq. (13) one must make a more general treatment. Following Ref. 12, the kinetic contribution to Eq. (13) is defined by the set valued function,

$$
\begin{gathered}
\frac{\partial \epsilon_{k}}{\partial n}=\left\{\hbar \omega_{c}(S+1 / 2)\right\} \quad \text { if } \quad \alpha \neq 0, \\
\frac{\partial \epsilon_{k}}{\partial n}=\left[\hbar \omega_{c}(S-1 / 2), \hbar \omega_{c}(S+1 / 2)\right] \quad \text { if } \quad \alpha=0,
\end{gathered}
$$

where $\{x\}$ denotes a single value and $\left[x_{1}, x_{2}\right]$ is a closed interval. The Euler equation can then be written as

$$
-V_{c}(r)+\mu_{c} \in \frac{\partial \epsilon_{k}(r)}{\partial n}
$$

where $V_{c}(r)$ includes the last five terms of the right-hand side of Eq. (15) and $\mu_{c}$ is constant along the radius.

For a fixed number of electrons and magnetic field, Lieb ${ }^{12}$ has proved that there is a unique non-negative density and a constant $\mu_{c}$ that satisfy Eq. (19).

In addition to this explanation, we went ahead with the specific realization of the kinetic term given by the fuction $C$ defined by Eq. (16) in order to obtain numerically the minimizer density. We define $\mu=\partial \epsilon / \partial n$ to distinguish it from the chemical potential $\mu_{c}$. The solution of Eq. (19) is obtained when the iterative process converges to a density that produces a constant value of $\mu$ within the regions where $\alpha$ $\neq 0$.

Physically this means that even though the energy of the last one-electron occupied state $\varepsilon_{F}$ is not the same over the whole dot, one can define a chemical potential. This is a consequence of dealing with strongly localized electrons for which Koopman's theorem, which identifies the $\mu_{c}$ constant with the Fermi energy, ${ }^{13}$ does not apply.

\section{B. The $\xi$ parameter}

The $\xi$ parameter has to be obtained from the minimization of the total energy against $\xi$. However, there are only three terms that explicitly depend on $\xi$ : the kinetic energy, the Coulomb exchange term, and the Zeeman splitting contribution. Apparently, the spin population obtained from only these three terms would correspond to an extended system free from any confining potential and therefore uninfluenced by $\omega_{0}$. However, the action of the confining potential on the 
spin population, which undoubtedly exists, is indirectly included via the local density $n(r)$ obtained by the solution of the whole Hamiltonian.

Therefore, the three terms that have to be minimized are

$$
\epsilon(\xi)=\epsilon_{k}+\epsilon_{e x}+\epsilon_{B},
$$

where $\epsilon_{k}$ from Eq. (2) can be written as

$$
\begin{gathered}
\epsilon_{k}=\frac{\hbar \omega_{c}}{2}\left\{n\left[1+S_{1}+S_{2}+\xi\left(S_{2}-S_{1}\right)\right]\right. \\
\left.-D\left[S_{1}\left(S_{1}+1\right)+S_{2}\left(S_{2}+1\right)\right]\right\}
\end{gathered}
$$

and where $\epsilon_{\mathrm{ex}}$ and $\epsilon_{B}$ are given by Eqs. (9) and (11), respectively. The value of $\xi$ that locally minimizes the energy [Eq. (20)] is not calculated from the condition of vanishing of the first derivative, but is explicitly obtained numerically from the variation of $\epsilon(\xi)$. The kinetic contribution increases monotonically with $\xi\left(S_{i}\right.$ are $\xi$ dependent) whereas the negative exchange contribution decreases also monotonically. The Zeeman splitting term gives a negligible decreasing $(g<0)$ contribution except for extremely high magnetic fields.

In the absence of any magnetic field, for high local density, the kinetic term is the dominant part and $\xi=0$ minimizes the energy (yielding a nonpolarized system). However, if the density is low (i.e., at the edge of the dot) the exchange contribution competes with the kinetic term and may produce a completely polarized system. The critical density that determines an abrupt change from fully polarized to nonpolarized systems (with zero magnetic field) is given by

$$
\epsilon\left(n_{c}, \xi=1\right)=\epsilon\left(n_{c}, \xi=0\right)
$$

or

$$
n_{c}=\left[\frac{8 \sqrt{2}(\sqrt{2}-1)}{3}\right]^{2} \frac{e^{4} m^{* 2}}{\hbar^{4} \varepsilon^{2} \pi^{3}}=2.44 \frac{e^{4} m^{* 2}}{\hbar^{4} \varepsilon^{2} \pi^{3}}
$$

(this means about 3 electrons within a dot of a radius of $R$ $=400 \AA$, or $n_{c}=6.82 \times 10^{10} \mathrm{~cm}^{-2}=0.0787 a_{B}^{*-2}$ for $m^{*}$ $=0.067 m_{e}$, and $\varepsilon=13.6$ ). Spontaneous spin polarization was previously reported, in the region of the saddle point in quantum point contacts as the electron density is lowered, using the spin-polarized density-functional theory of Kohn-Sham. ${ }^{11}$ This effect provided a qualitative explanation to conduction anomalies observed experimentally. ${ }^{14}$

Turning back to Fig. 1, which shows the Thomas-Fermi and the Landau-type kinetic contribution considered in Eq. (15), some structures can now be understood. At the center of the dot, the electron at the Fermi level lies at the seventh LL and falls into lower levels as the density decreases with $r$. An abrupt oscillation is apparent near the edge of the dot, where the density lies below the critical value. In this region, the system is suddenly fully polarized and the density of the greatest spin population suffers an abrupt increase promoting the last electron into higher levels.

The value of $\xi(n)$ that minimizes the energy is shown in Fig. 3 for two different values of the magnetic field. A nonzero magnetic field produces a critical density higher than the one given by Eq. (23). The oscillations of the $\xi$ parameter

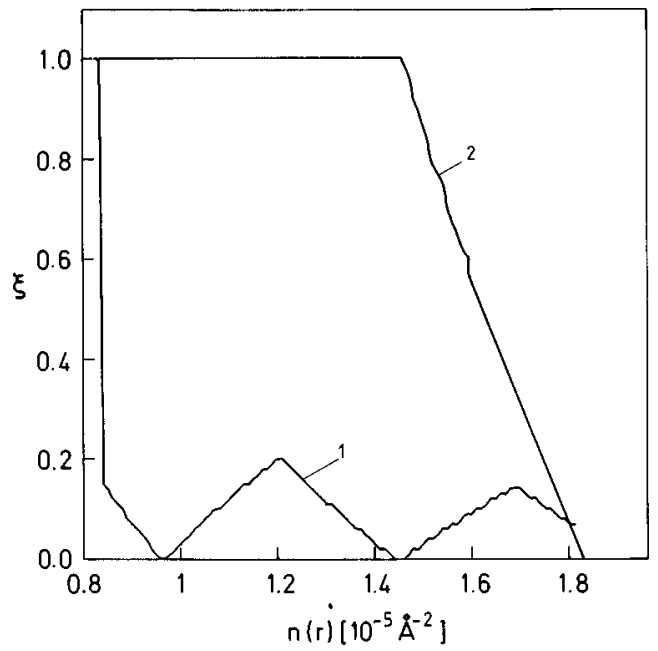

FIG. 3. Spin asymmetry parameter $\xi$ as a function of the electronic density. Curve 1 for $B=1 \mathrm{~T}$ and curve 2 for $B=6 \mathrm{~T}$.

produce the irregular structure of the kinetic and exchange contributions to the chemical potential as we show below.

The spin polarization of the entire dot is obtained from

$$
\xi_{\mathrm{eff}}=\frac{1}{N} \int \xi(r) n(r) d \vec{r}
$$

where $N$ is the total number of electrons.

\section{NUMERICAL CALCULATION}

To solve the Euler-Lagrange equation [Eq. (13)] we transform it into a fictitious Schrödinger equation in the following way: we add and subtract a Laplacian operator term:

$$
-a \frac{\Delta n}{n}+a \frac{\Delta n}{n}+\frac{\partial \epsilon_{t o t}}{\partial n}=\mu
$$

and substitute the last two terms on the left-hand side for their value at a given starting density $n_{0}$, obtaining

$$
-a \Delta n+\left[a \frac{\Delta n_{0}}{n_{0}}+\frac{\partial \epsilon_{\mathrm{tot}}}{\partial n_{0}}\right] n=\mu n .
$$

This can be written as

$$
h n=\mu n
$$

where

$$
h=-a \Delta+u_{0}, \quad u_{0}=a \frac{\Delta n_{0}}{n_{0}}+\frac{\partial \epsilon_{\text {tot }}}{\partial n_{0}}
$$

and where $n$ must satisfy the normalization condition

$$
\int n d \vec{r}=N .
$$

In this way Eq. (27) becomes an eigenvalue problem. As long as a convergent iterative procedure produces an output density equal to the input density coming from the previous step, the solution of Eq. (27) will be a solution of Eq. (13).

Now, we consider the equation 


$$
\frac{\partial n}{\partial \tau}=-h n
$$

where $\tau$ is a "fake time." 15 For positive eigenvalues, the net result of the time evolution of a linear combination of eigenfunctions is to enhance those components that have smaller eigenvalues. To understand what is going on, let the set of states $n_{\nu}$ be the eigenfunctions of the discrete operator $h$ with eigenvalues $\mu_{\nu}$. Since $h$ is Hermitian operator, the eigenvalues are real, the eigenvectors can be chosen to be orthonormal, and an expansion of $n$ on this basis at any time is given by

$$
n_{j}=\sum_{\nu} \alpha_{\nu}^{j} n_{\nu}
$$

where time evolution is represented by steps of $\Delta \tau$ for $j$ $=0,1,2, \ldots$. On the other hand, the exact time evolution of $n$ is given by

$$
n_{j}=e^{-h j \Delta \tau} n_{0}
$$

in such a way that each component of the linear combination should evolve as

$$
\alpha_{\nu}^{j}=e^{-\mu_{\nu} j t} \alpha_{\nu}^{0}
$$

That is to say, components with larger eigenvalues disappear more rapidly, leaving the eigenstate of lowest energy as the dominant contribution for sufficiently long time evolution.

As long as $\Delta \tau$ is chosen to be small enough, Eq. (32) can be written as

$$
n_{1}=(1-\Delta \tau h) n_{0}=\frac{1-(\Delta \tau / 2) h}{1+(\Delta \tau / 2) h} n_{0}
$$

after the first step [to order $\left.(\Delta \tau)^{2}\right]$, or

$$
\left(1+\frac{\Delta \tau}{2} h\right) n_{1}=\left(1-\frac{\Delta \tau}{2} h\right) n_{0} .
$$

Finally, if the right-hand side is fully calculated at the initial density $n_{0}$, then we obtain

$$
\left(1+\frac{\Delta \tau}{2} h\right) n_{1}=\left(1-\frac{\Delta \tau}{2} \frac{\partial \epsilon_{\mathrm{tot}}}{\partial n_{0}}\right) n_{0} .
$$

If an iterative calculation is performed, normalizing $n_{j}$ after each step, the convergent process must evolve to the ground-state density of the system.

The space derivatives are approximated by finite differences on a uniform lattice of $N+1$ points having spacing $d$ $=1 / N$, therefore transforming Eq. (36) into an $(N+1) \times(N$ $+1)$ system of equations that can be solved by inverting the matrix $[1+(\Delta \tau / 2) h]_{i j}, i, j=1,2, \ldots N+1$.

Although this numerical procedure is straightforward, it involves a few subtleties. The parameter $a$ must be chosen in such a way that $a \Delta n_{0} / n_{0}$ and $\partial \epsilon_{\text {tot }} / \partial n_{0}$ are of the same order of magnitude and $\Delta \tau$ must guarantee that the approximations made in the expansions of Eq. (34) are valid. Moreover, it is sometimes necessary to average the last two densities to generate the next.
The appropriate boundary conditions for our problem are the cancellation of the first-order derivatives at $r(1)$ and $r(N+1)$ and, must be implemented in the first and last rows of the $(N+1) \times(N+1)$ matrix in Eq. (36) on the left-hand side and in the first and last values of the column on the right-hand side.

In some cases, the convergence of the numerical process turns out to be quite sensitive to the initial input density. As a rule of thumb, it is convenient to begin with an electronic density confined within the classical radius $R$ given in the next section by Eq. (39), and which approximately reproduces the value $n(0)$ given by Eq. (38).

\section{RESULTS}

Some test calculations will first be discussed. They are of some interest in their own right, besides providing a check on the method and the numerical calculation. We will concentrate on three such tests:

(1) Semiclassical model. An analytical solution of the g.s. density can be obtained ${ }^{16,17}$ if the Hamiltonian contains only two terms, the direct Coulomb contribution and the confining potential. The solution is given by a "dome", density, given by

$$
n(r)=n(0) \sqrt{1-\frac{r^{2}}{R^{2}}},
$$

where

$$
n(0)=\frac{3 N}{2 \pi R^{2}}
$$

and where a relation between the three parameters, $\omega_{0}, R$, and $N$ must hold:

$$
\omega_{0}=\sqrt{\frac{3 \pi N e^{2}}{4 \varepsilon m^{*} R^{3}}} .
$$

In Fig. 4 the two terms that enter the Euler equation are displayed (as "dir"' and "conf'"). The Coulomb potential created by the electronic density and the confining potential cancel each other out, producing a constant chemical potential throughout the dot (" $\mu$ '"). These two terms are the main ones in the general case, which includes all the terms of the Hamiltonian, as is verified below, and as a consequence, the effective potential seen by an electron inside the dot will always be quite flat. To see the relative importance of each of the terms in the full Hamiltonian, in Fig. 4 we also show their contributions separately using the dome density as a first approximation. It must be stressed that the dome density is not a solution of the whole Hamiltonian and that the " $\mu$ ", curve contains only the "dir"' and "conf"' terms.

Although the model used has certain similarities with the Thomas-Fermi model, particularly in the way the system is inspected locally, point by point, there are significant differences. As in the Thomas-Fermi model, one has to worry about the space variations in the fields felt by the electrons. The models are justified only in the case where the potentials are flat enough to consider free electrons locally, in such a way that the local effect of the potentials is to change the 


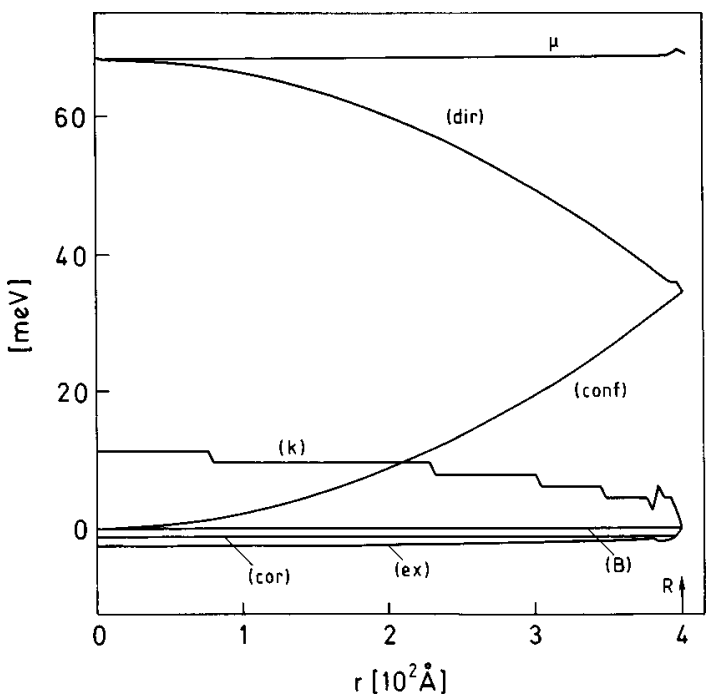

FIG. 4. Direct Coulomb (dir) and confining potential (conf) contributions to the Euler equation along the radius using the dome density for 10 electrons and $R=400 \AA, g=-0.44, m^{*}$ $=0.067 m_{e}, \hbar \omega_{0}=2.6 \mathrm{meV}$ and $\epsilon=12.4 . \mu$ being the chemical potential for this two terms. Contributions to the Euler equation from the kinetic $(k)$, correlation (cor), exchange (ex) and Zeeman splitting $(B)$ terms are also displayed.

zero of energy. Under this hypothesis, the semiclassical Thomas-Fermi kinetic energy is calculated assuming a continuous density of states and full occupation of each level (the total number of electrons is always an even number). In contrast, in our model the kinetic contribution is the exact quantum contribution from a free system of electrons, in which the discrete energy levels with finite degeneracy are considered for each spin state. In order to recover the Thomas-Fermi result as a limit, one must reduce the energy gaps to zero (decreasing the magnetic field) and consider all levels as being fully occupied.

One consequence of our model is the appearance of strong variations in the kinetic contribution to the Euler equation within short distances (see Figs. 1 and 4). However, this effect does not violate our initial hypothesis concerning the smoothness of the potentials, which justifies the free electron assumption.

Besides this argument, it is true that the use of wave functions would smooth the abrupt variations of the kinetic term used to generate new densities after each iteration. To see if it had any effect on the final converged density, we convoluted $\epsilon_{k}(r)$ within each iteration, using a Gaussian function with a width typical of a single electron wave function of the Laguerre type [Eq. (40)]. However, within the numerical precision considered, we could find no differences.

(2) If in contrast, within the Hamiltonian we include only the kinetic term (including a nonvanishing magnetic field) and the confining potential, and as such we define a system of independent electrons, the analytical solution for the eigenfunctions is known to be given by Laguerre polynomials $L_{n}^{|l|}{ }^{18}$

$$
\phi_{n l}(r, \theta)=\sqrt{\frac{n !}{2 \pi(n+|l|) !}} \frac{1}{\lambda} e^{-i l \theta} u^{|l| / 2} e^{-u / 2} L_{n}^{|l|}(u),
$$
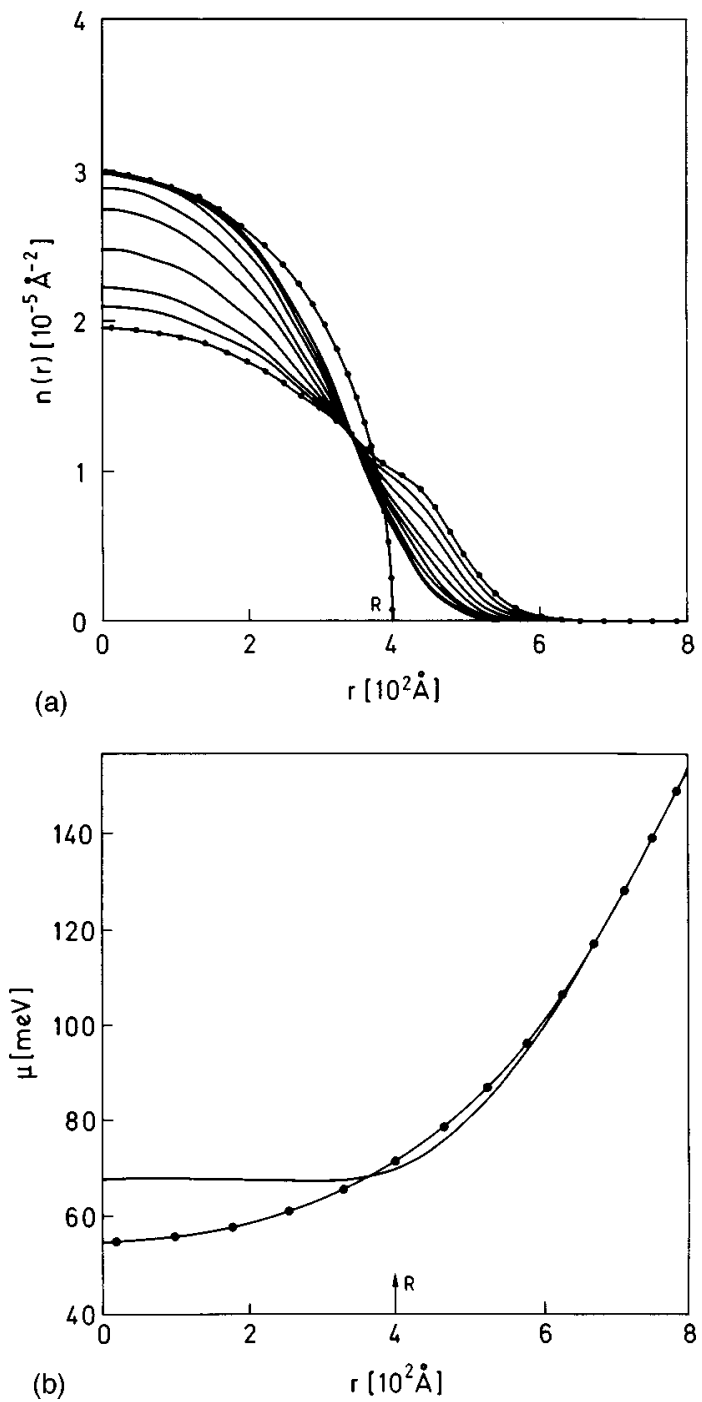

FIG. 5. (a) Succesive iterations (solid lines) generated from a semiclassical model Hamiltonian (see text). The lower dotted curve is the starting density $n_{0}$ and the upper dotted curve the exact dome solution. (b) Chemical potentials for $n_{0}$ ( dotted curve) and the last iteration (full curve).

where $n=0,1,2 \ldots, l=0, \pm 1, \pm 2, \ldots$,

$$
\lambda^{2}=\frac{\hbar}{m^{*} \tilde{\omega}}, \quad \tilde{\omega}=\sqrt{\omega_{c}^{2}+4 \omega_{0}^{2}}
$$

and $u=r^{2} /\left(2 \lambda^{2}\right)$.

Since the Laguerre polynomials are well behaved at the boundaries, we selected these wave functions as a standard way for generating the starting density within the general iterative procedure.

As a second test, we began with $n_{0}$ generated by Laguerre polynomials and looked for the solution of the semiclassical model, that is, when only the Coulomb and confining potential terms are included in the Hamiltonian. Taking the first 50 wave functions of lowest energy to produce the starting density $n_{0}$ (normalized to $N$ electrons), the iterative procedure produces the density shown in Fig. 5(a). The lower dotted curve is the starting density $n_{0}$, the upper dotted curve is the exact dome density included for comparison, and the con- 


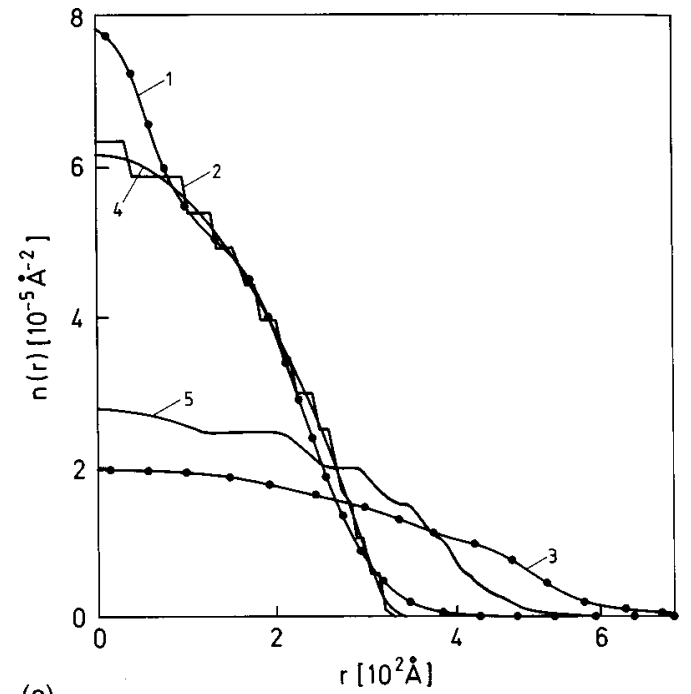

(a)

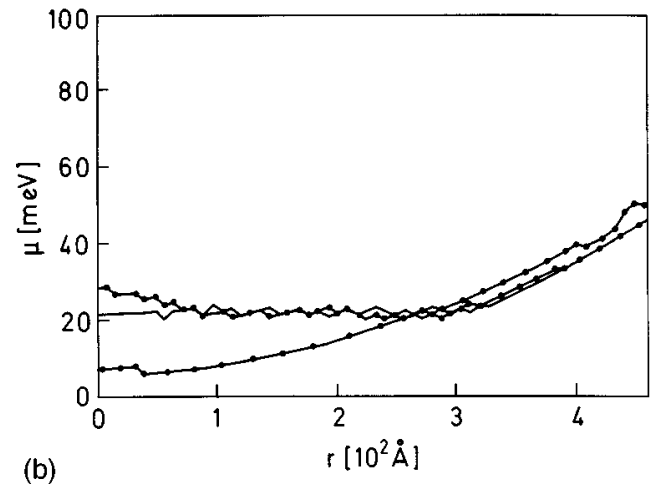

FIG. 6. (a) Electronic density of a 10 electron dot for $B$ $=1 \mathrm{~T}, \epsilon=12.4$ and $\hbar \omega_{0}=2.6 \mathrm{meV}$. For a noninteracting system: curve 1 is the exact solution, added for comparison, curve 3 is the starting density $n_{0}$, curve 2 is the numerical result and curve 4 is the Thomas-Fermi approximation. Curve 5 is the numerical result that includes electron-electron interaction. (b) Chemical potentials for the first three densities: lower dotted curve is for $n_{0}$, upper dotted curve is for the exact solution, and the full curve is for the numerical result.

tinuous lines give succesive iterations. However, we were unable to arrive at the exact solution because of the different boundary conditions at $R$. The exact solution has an infinite derivative whereas the iterative procedure produces densities of vanishing first derivative at the edge of the dot. However, the last iteration gives a good approximation to the Euler equation as shown in Fig. 5(b).

(3) For the last test, a system of 10 independent electrons was considered. The result obtained is shown in Fig. 6(a) where the upper dotted curve labeled 1 and included for comparison is the exact solution built up from the first five functions [given by Eq. (40)] of the lowest energy (each level filled with two electrons of spin up and down) and the lower dotted curve labeled 3 is the starting density. The result of the iteration procedure is shown as a solid line labeled 2 . It must be realized that the probability of finding an electron at $r$ is given by $r n(r)$. Multiplication by $r$ would diminish the differences, enhanced in the representation of $n(r)$. There is, however, a remarkable coincidence particularly if we consider that it is a system of only 10 electrons within a model that tends to the exact quantum solution for $N \rightarrow \infty$. ${ }^{12}$ Within this calculation, an unpolarized system with constant $\xi=0$ was considered (there is no exchange interaction and the magnetic field considered is small). The starting density was the same as that used above, generated from 50 Laguerre functions and normalized to 10 electrons.

The chemical potentials are shown in Fig. 6(b). Due to the different treatment of the kinetic term the exact density is not the solution to our Euler equation, and the iterations converge to a different density, which nevertheless is relatively close to the exact solution.

Our result produces a succession of small steps that follow the semiclassical Thomas-Fermi result displayed by curve 4 and obtained from the replacement of the kinetic term by the expression $\hbar^{2} \pi n(r) / m^{*}$. The agreement between the Thomas-Fermi profile and the exact solution in most of the $r$ range is due to the small influence that the magnetic field has at this relatively high density.

This result would suggest that the inclusion of the LL type kinetic term does not produce a great improvement in the results if compared to those obtained from the semiclassical Thomas-Fermi model. Nonetheless, a qualitatively different result is obtained when an electron-electron interaction is considered including not only the direct Coulomb term but also the exchange interaction. The formation of plateaus in the density profile is shown (curve 5) as a consequence of electronic interaction. The width of the plateaus changes as the magnetic field increases, as is shown below. It must be stressed that the steps of curve 2 are a direct manifestation of the LL's whereas the plateaus of curve 5 result from the interplay between kinetic and Coulomb interaction.

Before discussing the addition spectrum calculation, it proved both useful and instructive to analyze the evolution of the g.s. electronic density and the g.s. energy with the magnetic field. From now on the parameters used are those of the experiment reported in Ref. 2: $g=-0.44, m^{*}$ $=0.067 m_{e}, \quad N=39$ (the number of electrons), $\hbar \omega_{0}$ $=1.6 \mathrm{meV}$, and $\epsilon=13.6$. In Fig. 7 the evolution of the profile density is shown as a function of the magnetic field (from 0.4 to $2.9 \mathrm{~T}$ ).

As the magnetic field changes, the periodic formation of density plateaus becomes visible. At $B=1.1 \mathrm{~T}$ two constant density regions are produced at around $r=7.5 a_{B}^{*}$ and at $r$ $=14 a_{B}^{*}$. These disappear for greater values of $B$, appearing again for $B=2.1 \mathrm{~T}$ at around $r=9.3 a_{B}^{*}$.

A Lang-Kohn type calculation produces Friedel oscillations in the density profiles that mask the plateaus, as is the case in Refs. 19 and 20 where moreover, a different density functional for the exchange interaction is used.

It is also apparent in Fig. 7 that for very small or very large values of $B$, the density resembles that of the classical dome, although the energetic structure of the dots differs greatly. For high $B$ the kinetic term tends towards an $r$ independent value given by the first LL, while for very low values of $B$ the kinetic term tends to the semiclassical value $\pi n(r)$.

The competition between the tendency for the density to increase at the center of the dot as the magnetic field increases and for the density to decrease as a result of the Coulomb repulsion produces roughly the same density at $r$ $=0$ for all the values of $B$. 


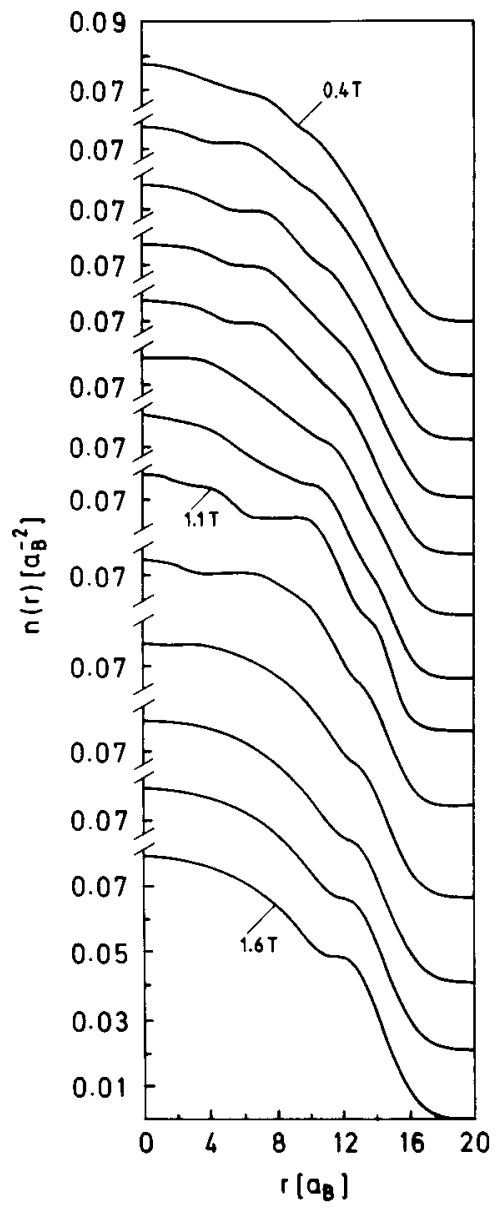

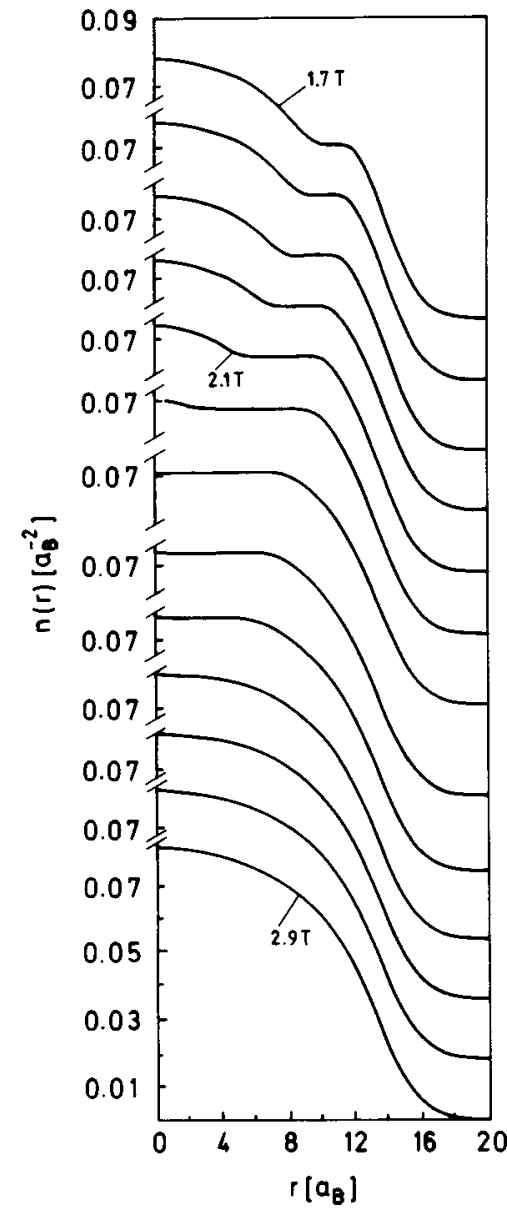

FIG. 7. Evolution of the density profile with the magnetic field for the 39 electrons quantum dot from $B=0.4 \mathrm{~T}$ to $2.9 \mathrm{~T}$ by steps of $0.1 \mathrm{~T}$. From now on, the parameters proposed in Ref. 2 have been used, i.e., $m^{*}=0.067 m_{e}$ with $m_{e}$ electron mass, $\epsilon=13.6$, which give a Bohr radius $a_{B}^{*}=\hbar^{2} \epsilon / m^{*} e^{2}=107.42 \AA$ and $\hbar \omega_{0}=1.6$ meV.
The plateaus are related to the formation of incompressible circular regions within the $\operatorname{dot}^{21}$ where LL's are fully occupied. This can be seen in Fig. 8(a), in which the local value of the filling factor for $B=1.1 \mathrm{~T}$ is displayed. Within these regions the electrons show no mobility and their effective potential is not fully screened, as can be verified in Fig. 8(b) where the effective potential, obtained from the lefthand side of Eq. (13) by the subtraction of the kinetic term, is also shown. In contrast, within the compressible regions, the effective potential is constant. Here the metallic character screens the fields and there is no net force on the charges. Within the incompressible regions, the gradient of the effective potential has a nonzero value and the equilibrium is obtained by discrete changes in the kinetic energy, producing wiggles in $\mu$ as shown in Fig. 8(b).

As the magnetic field increases, only one strongly degenerate LL is occupied, and the full dot acquires a metallic character producing a constant function $\mu$ throughout the dot. Much stronger fields (about $30 \mathrm{~T}$ ) would produce new effects related to the fractional quantum Hall regime.

Figure 9 shows the variation of the total energy [Eq. (1)] with $B$. The different contributions to the energy are included, at different scales. The Zeeman splitting term is not included as it is a negligible monotonous decreasing contribution (of order $10^{-2} \mathrm{meV}$ ).

Over all the values of $B$, partial cancelation between the direct Coulomb term and the confining potential takes place, as was the case in the semiclassical test. To understand this behavior, it is easier to follow the evolution of the mean radius of the density distribution, defined as the integral of $r n(r)$ and not included in the figures, since it has virtually the same dependence on $B$ as the confining potential contribution, proportional to the integral of $r^{2} n(r)$ and displayed in Fig. 9(b) (as $E_{\text {conf }}$ ). As the mean radius increases, the confining potential contribution also increases. In contrast, the expansion of the electronic density diminishes the direct Coulomb repulsion at a very similar rate. This competition flattens their contribution to the total energy. Although most of the total energy comes from the electrostatic terms (direct Coulomb and confining potential), their partial cancelation produces the following effect: the variation of the total energy with $B$ is in fact quite sensitive to the nondominant contributions, the exchange term for weak $B$ and the kinetic term for strong magnetic fields. A type of oscillatory behavior in the mean radius was previously obtained in Ref. 19. These authors relate the periodic oscillations to changes in the angular momentum. With increasing magnetic field, the density moves inward becoming more highly localized around the origin, provided the angular momentum remains constant. This leads to an increase in electrostatic energy, which is suddenly released when the angular momentum changes its value and the density peak moves outward. This pattern is repeated every time the orbital angular momentum increases.

For large values of $B$, the kinetic term (preportional to $\omega_{c}$ ) increases in value determining the variation of the total energy with $B$. The total energy behaves as the energy of an independent particle system in a metalliclike regime, where only the kinetic energy is important. In contrast, the Coulomb interaction has an inert role, having been frozen. This 

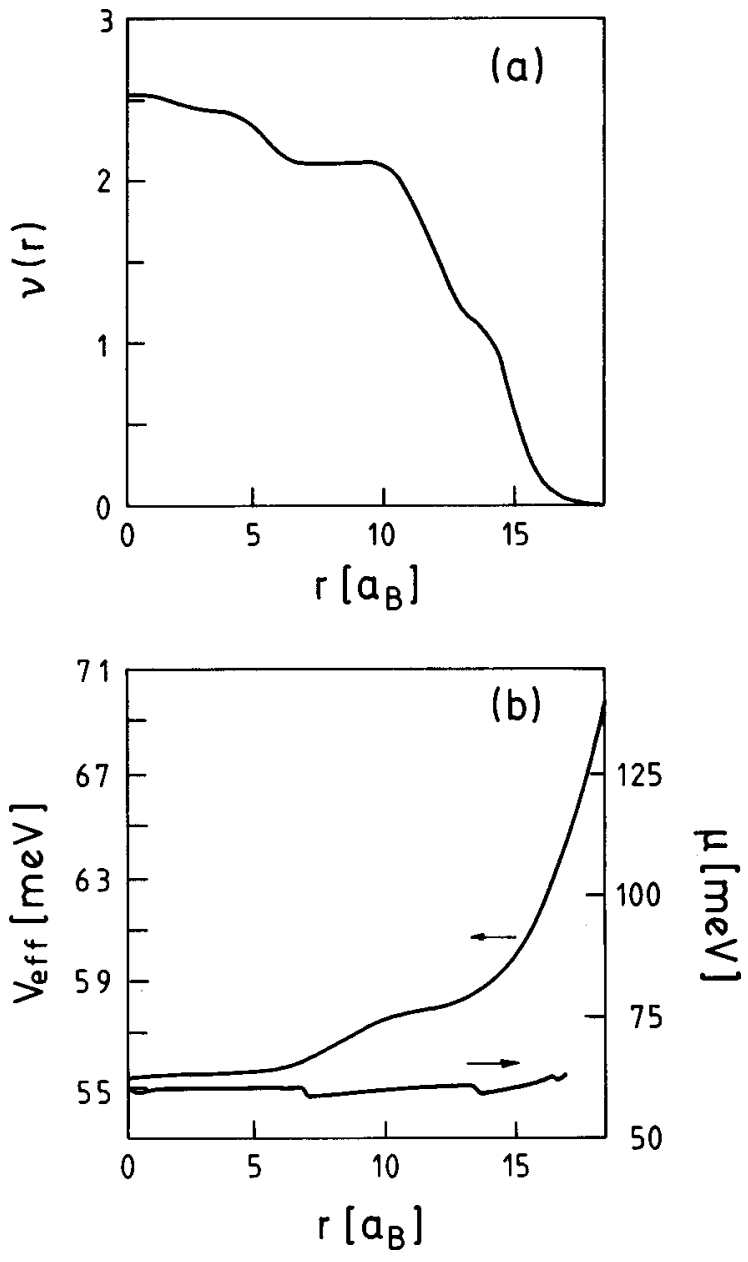

FIG. 8. (a) Local filling factor along the radius. (b) Left axis labeling: effective potential and right axis labeling: chemical potential. In all cases the density displayed in Fig. 7 at $B=1.1 \mathrm{~T}$, is used.

is the case for strongly correlated systems (for example, a high density electron gas in which the electron-electron interaction is nearly completely screened).

Figure 10 shows the addition spectrum $E(N)-E(N-1)$ $=\mu_{c}$ for different magnetic fields. The spectrum shows a structure superimposed on the general increase with $B$, which has been interpreted in different ways within the literature.

As suggested by the independent electron result, the kink of $\mu_{c}(B)$ at about $1.9 \mathrm{~T}$ in the experimental data has been related to filling factor $\nu=2$ : the first LL is fully occupied and the spin population is symmetric. From 2 to $3 \mathrm{~T}$, the small wiggles would be produced by successive jumps of electrons from inside the dot to the outside as a result of Coulomb electrostatic repulsion, with a simultaneous spin flipping induced by the exchange interaction. This process ends when the compact droplet with $\nu=1$ is formed.

Our results suggest a different explanation. For the low value of $\hbar \omega_{0}=1.6 \mathrm{meV}$ proposed in Ref. 2, the electronic density $n(r)$ lies below the critical value [see Eq. (23)] except at $r$ close to the origin, producing an effective asymmetry parameter very close to one [see Eq. (24)], i.e., a fully polarized system for all the values of the magnetic field. In other words, the exchange interaction has promoted the

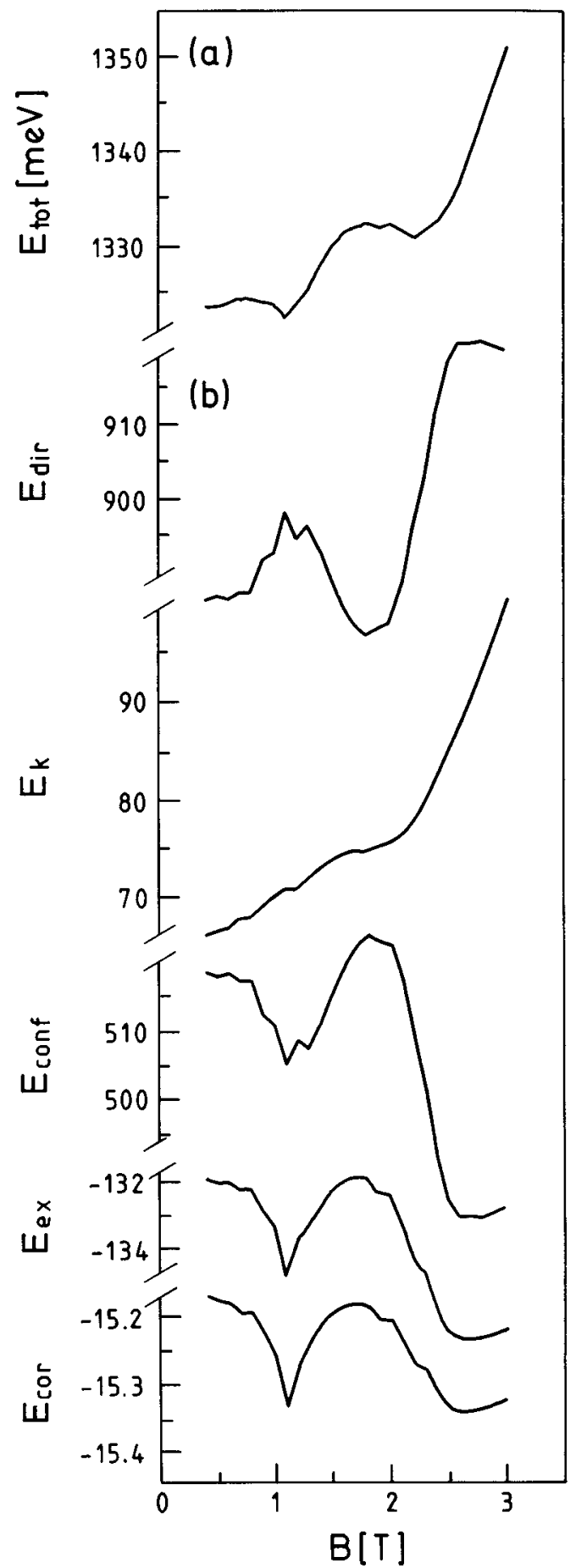

FIG. 9. (a) Variation of the total energy [Eq. (1)] as a function of the magnetic field. (b) Different contributions to the total energy displayed on different scales.

spin-up (or down depending on the sign of the Landé factor) states to much higher energies, and different LL are closer in energy than different spin states. A higher value of $\hbar \omega_{0}$ would produce higher densities and the possibility of successive variations induced by $B$, as seems to be reflected by the experimental data. That is to say, we suggest that these experimental data could not have been produced by such a low value of $\hbar \omega_{0}$.

Besides this conclusion, the curve displayed in Fig. 10 can be interpreted for a fully polarized system in the follow- 

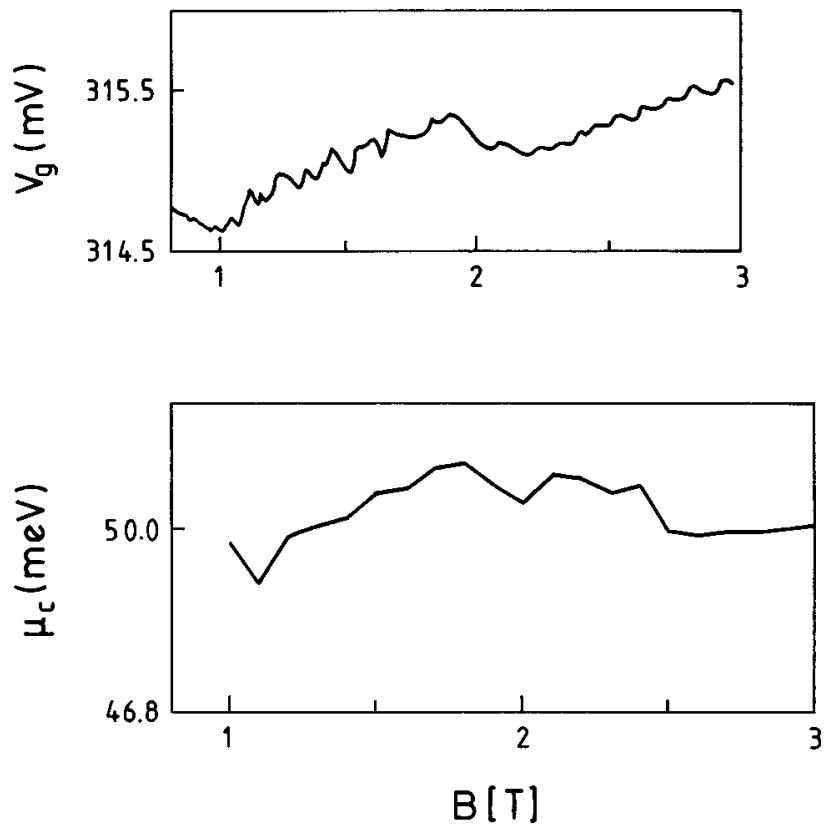

FIG. 10. Chemical potential as a function of the magnetic field. Experimental results of the back-gate voltage from Ref. 2 are also displayed.

ing way: at first, it is necessary to stress that the definition of filling factor in the 2D independent system of electrons cannot be extrapolated directly to a quantum dot of interacting electrons. As a consequence of the space localization of the electrons in different regions (compressible or incompressible) and the electron interaction, the filling factor becomes a local function. In Fig. 11 we display its value at the center of the dot, and though its variation with $B$ gives useful information, its significance has limitations.

The $\mu_{c} / B$ curve is a consequence of the variation of $E(N)$ with $B$. The minima of $E(N)$ at about $B=1.1$ and $B$

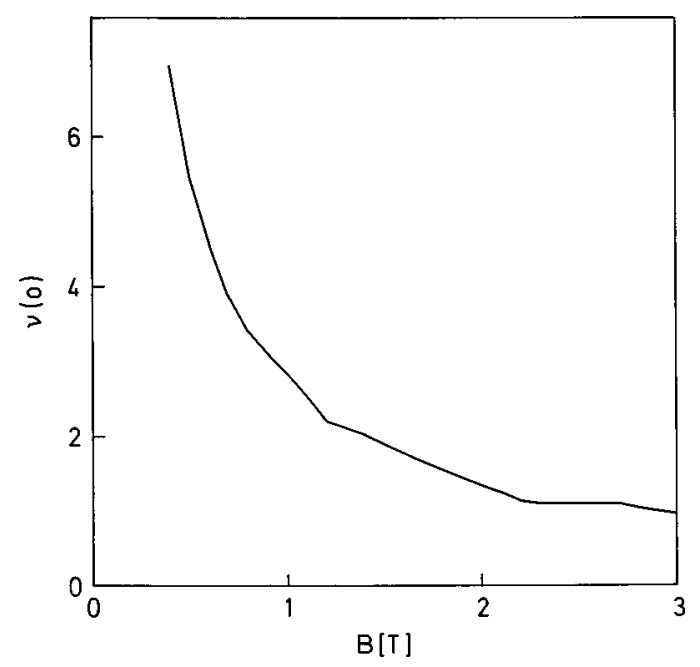

FIG. 11. Filling factor at the center of the dot as a function of the magnetic field.

$=2.2 \mathrm{~T}[$ see Fig. 9(a)] are related to the appearance of marked plateaus in the profile density at these same values of $B$ and also related to $\nu(0)=2$ and 1 , respectively. It must be stressed, however, that $\nu=2$ in our result means that the two first LL are occupied by electrons of the same spin state. As mentioned previously, the contribution of the kinetic term and the exchange interaction determines the final shape of the $\mu_{c} / B$ curve.

\section{ACKNOWLEDGMENTS}

We gratefully acknowledge J. Martorell, L. Brey, and C. Tejedor for useful discussions. This study has been performed under Grant No. PB95-1249 from CICYT, Spain, and Grant No. 1996SGR from Generalitat of Catalunya.
${ }^{1}$ L. Jacak, P. Hawrylak, and A. Wójs, Quantum Dots (SpringerVerlag, Berlin, 1998).

${ }^{2}$ P. L. McEuen, E. B. Foxman, J. Kinaret, U. Meirav, M. A. Kastner, N. S. Wingreen, and S. J. Wind, Phys. Rev. B 45, 11419 (1992).

${ }^{3}$ I. K. Marmorkos and C. W. J. Beenakker, Phys. Rev. B 46, 15 562 (1992).

${ }^{4}$ R. C. Ashoori, H. L. Stormer, J. S. Weiner, L. N. Pfeiffer, K. W. Baldwin, and K. W. West, Phys. Rev. Lett. 71, 613 (1993).

${ }^{5}$ D. B. Chklovskii, K. A. Matveev, and B. I. Shklovskii, Phys. Rev. B 47, 12605 (1993).

${ }^{6}$ M. Stopa, J. P. Bird, K. Ishibashi, Y. Aoyagi, and T. Sugano, Phys. Rev. Lett. 76, 2145 (1996).

${ }^{7}$ J. J. Palacios, L. Martín-Moreno, G. Chiappe, E. Louis, and C. Tejedor, Phys. Rev. B 50, 5760 (1994).

${ }^{8}$ M. Abramowitz and I. A. Stegun, Handbook of Mathematical Functions (Dover, New York, 1972).

${ }^{9}$ F. Stern, Phys. Rev. Lett. 30, 278 (1973).

${ }^{10}$ C. K. Wang and K. F. Berggren, Phys. Rev. B 57, 4552 (1998).
${ }^{11}$ J. Wang, Y. Wang, and H. Guo, J. Appl. Phys. 75, 2721 (1994).

${ }^{12}$ E. H. Lieb, J. P. Solovej, and J. Yngvason, Phys. Rev. B 51, 10 646 (1995).

${ }^{13} \mathrm{~W}$. Kohn and P. Vashishta, in Theory of the Inhomogeneous Electron Gas, edited by S. Lundqvist and N. H. March (Plenum Press, New York, 1983).

${ }^{14}$ K. J. Thomas, J. T. Nicholls, M. Y. Simmons, M. Pepper, D. R. Mace, and D. A. Ritchie, Phys. Rev. Lett. 77, 135 (1996).

${ }^{15}$ S. E. Koonin and D. C. Meredith, Computational Physiscs (Addison-Wesley, New York, 1990).

${ }^{16}$ V. Shikin, S. Nazin, D. Heitmann, and T. Demel, Phys. Rev. B 43, 11903 (1991).

${ }^{17}$ Z. L. Ye and E. Zaremba, Phys. Rev. B 50, 17217 (1994).

${ }^{18}$ V. Gudmundsson and R. R. Gerhardts, Phys. Rev. B 43, 12098 (1991).

${ }^{19}$ M. Ferconi and G. Vignale, Phys. Rev. B 50, 14722 (1994).

${ }^{20}$ E. Lipparini, N. Barberan, M. Barranco, M. Pi, and L1. Serra, Phys. Rev. B 56, 12375 (1997).

${ }^{21}$ A. M. Chang, Solid State Commun. 74, 871 (1990). 